\title{
Outcomes of 596 Advanced Gastric Cancer Patients with Different Numbers of Chemotherapy Lines: The More Chemotherapy Lines, the Better Survival
}

This article was published in the following Dove Press journal: Cancer Management and Research

\author{
Li Sun ${ }^{1} *$ \\ Huijun Wangl,* \\ Zhen Liu' \\ Ying Meng' \\ Meiqing Qiu ${ }^{2}$ \\ Yafei Ju' \\ Shu Zhang $\mathbb{D}^{\prime}$
}

'Department of Gastroenterology, Shandong Cancer Hospital and Institute, Shandong First Medical University and Shandong Academy of Medical Sciences, Jinan, People's Republic of China; 2Department of Oncology, Zaozhuang Municipal Hospital, Zaozhuang, People's Republic of China

*These authors contributed equally to this work
Correspondence: Shu Zhang

Department of Gastroenterology, Shandong Cancer Hospital and Institute, Shandong First Medical University and Shandong Academy of Medical Sciences, Jinan, People's Republic of China Tel/Fax +86-053I-67626836

Email zhangshu@csco.org.cn
Objective: Many large-sample prospective randomized clinical trials investigating advanced gastric cancer (AGC) have confirmed the survival advantages of first-line, secondline, or third-line chemotherapy compared with their respective control groups. However, due to the ethical concerns of prospective clinical trials, it is impossible to conduct a randomized comparative study of patients who do not receive chemotherapy and those who receive a second-line or above chemotherapy. Few research reports have addressed the relationship between the number of chemotherapy lines and overall survival (OS) in patients with AGC. In the present study, we analyzed the impact of the number of chemotherapy lines on OS in AGC patients using real-world data.

Patients and Methods: This study collected the medical records of patients with AGC diagnosed at Shandong Cancer Hospital from December 2007 to December 2017. According to the treatment received, AGC patients were divided into groups that did not receive chemotherapy, those who received only 1 line, 2 lines, or 3 lines and above. Kaplan-Meier analysis was used to assess patient survival.

Results: A total of 596 AGC patients were included in this study. The following patients were enrolled: 0 lines (did not receive chemotherapy), 77 (12.9\%); 1 line, 235 (39.4\%) patients; 2 lines, 185 (31.1\%) patients; and $\geq 3$ lines 99 (16.6\%) patients. OS was significantly correlated with the number of chemotherapy lines $(\mathrm{P}<0.001)$, with a median OS from diagnosis of 3.3, 8.6, 15.6, and 21.0 months for patients receiving $0,1,2, \geq 3$ lines of chemotherapy, respectively.

Conclusion: This study showed that the more chemotherapy lines AGC patients received, the longer the OS. This study not only confirmed the impact of chemotherapy lines on OS but it also supplements the results of prospective clinical trials that cannot be completed due to the ethical implications.

Keywords: advanced gastric cancer, number of chemotherapy lines, overall survival, real world data

\section{Introduction}

Gastric cancer is the fifth most common malignancy and the third leading cause of cancer-related death in the world. East Asia has the highest incidence and mortality rate from gastric cancer. ${ }^{1}$ In 2018, there were 450,000 new cases and 390,000 deaths in China. ${ }^{2}$ The prognosis of gastric cancer is very poor. Approximately $80 \%$ of patients are at an advanced stage when they are diagnosed, and the 5-year overall survival (OS) rate is less than $25 \%^{3}$

Comprehensive medical treatment is the main treatment approach for advanced gastric cancer (AGC). Many clinical trials have confirmed that the median OS of 
patients who have only received best supportive care (BSC) is $3-5$ months, ${ }^{3}$ while the first-line combination chemotherapy based on fluoropyrimidine or platinum is 8.8-10.0 months. ${ }^{4,5}$ The median OS of second-line chemotherapy (taxanes or irinotecan) is 5.2-7.7 months, ${ }^{6-8}$ and that of third-line treatment (including chemotherapy and targeted therapy) is 4.0-6.5 months. ${ }^{9,10}$ Many largesample prospective randomized clinical trials of AGC have confirmed the survival advantages of first-line, second-line, or third-line chemotherapy compared with their respective control groups. ${ }^{10-12}$ However, due to the ethical factors involved with prospective trials, it is impossible to conduct a randomized comparative study of patients who do not receive chemotherapy and those who receive a second-line and above. There are few research reports on the relationship between the number of chemotherapy lines and OS in AGC patients. This study investigated the impact of the number of chemotherapy lines on the survival of AGC patients using real-world data.

\section{Patients and Methods}

\section{Patients}

We screened patients with AGC who were diagnosed and treated at Shandong Cancer Hospital from December 2007 to December 2017. The inclusion criteria for study participation were as follows: (1) age 18 years or above; (2) diagnosis of metastatic or recurrent, unresectable gastric, or gastroesophageal junction adenocarcinoma confirmed by histology; (3) no other prior or concomitant malignancy; (4) expected survival more than 3 months; and (5) complete medical records. The exclusion criteria were the following: (1) expected survival less than 3 months; (2) incomplete medical records; and (3) severe heart, cerebrovascular, liver, kidney or hematopoietic system disease. This retrospective study was approved by the Institutional Review Board of our institution (IRB No. 201909002). Written informed consent was waived, given the analysis was based on data retrieved from electronic medical records with anonymous selection. We commit to strictly complying with data confidentiality principles, and the Declaration of Helsinki.

\section{Methods}

Patient medical records were obtained through the hospital information system. Information such as age, sex, time of diagnosis, ECOG performance status, tumor location, metastasis location, treatment, and overall best response were recorded in detail. According to the treatments received, AGC patients were divided into the following groups: no chemotherapy, only 1 line of chemotherapy, only 2 lines of chemotherapy, or 3 or more lines of chemotherapy. The primary endpoint of this study was OS, which was calculated from the date of AGC diagnosis to date of death. Patients surviving at the time of the data collection were censored at the date of last contact. Patients who were reported to have died, but for whom no date of death was available, were assumed to have died on the date of last contact.

\section{Statistical Analysis}

OS was estimated according to the Kaplan-Meier method, and the statistical significance of differences in survival curves between the 4 groups was tested with a Log rank test. $\mathrm{P}$ values $<0.05$ were considered statistically significant. All P values corresponded to 2-sided tests. All Statistical analyses were conducted using the SPSS v22.0 software package (SPSS, Chicago, IL, USA).

\section{Results}

\section{Patient Characteristics}

A total of 596 patients were identified, of which 427 (71.6\%) were men and 169 (28.4\%) were women. The median age at diagnosis was 59 years (range, 20-85 years). ECOG performance status at initial treatment was 0 for $36(6.1 \%), 1$ for 458 (76.8\%), and 2 for $102(17.1 \%)$ patients. The most common primary tumor locations were the antrum and pylorus (45.8\%), followed by the fundus and the body of the stomach $(28.7 \%)$. The most common sites of metastasis were the lymph nodes $(68.0 \%)$, peritoneum $(44.6 \%)$, and liver $(34.2 \%)$. The patient characteristics are summarized in Table 1.

\section{Treatment}

Of the 596 patients in this study, 519 patients had received chemotherapy and 77 patients $(12.9 \%)$ did not receive chemotherapy. Among 519 patients who had received firstline chemotherapy, $138(26.6 \%)$ received a triplet chemotherapy regimen, which included predominantly fluoropyrimidine/platinum doublets with the addition of either taxanes or an anthracycline; 327 (63.0\%) received doublet therapy, predominantly a fluoropyrimidine/platinum doublet; and 54 (10.4\%) received single-agent treatment. Of the 519 patients, 284 (54.7\%) subsequently received second-line treatment. Of these 284 patients, 24 
Table I Patient Characteristics $(n=596)$

\begin{tabular}{|l|l|}
\hline Characteristics & Patients, $\mathbf{n}$ (\%) \\
\hline Sex & \\
Male & $427(71.6)$ \\
Female & $169(28.4)$ \\
\hline Age at diagnosis, years & \\
$20-39$ & $55(9.2)$ \\
$40-49$ & $111(18.6)$ \\
$50-59$ & $137(23.0)$ \\
$60-69$ & $199(33.4)$ \\
$\geq 70$ & $94(15.8)$ \\
\hline ECOG performance status & \\
0 & $36(6.1)$ \\
I & $458(76.8)$ \\
2 & $102(17.1)$ \\
\hline Tumor location & \\
Antrum and pylorus & $273(45.8)$ \\
Fundus and body & $171(28.7)$ \\
Gastric cardia & $89(14.9)$ \\
Esophagogastric junction & $42(7.1)$ \\
Whole stomach & $21(3.5)$ \\
\hline Metastatic site & \\
Lymph nodes & $405(68.0)$ \\
Peritoneum & $266(44.6)$ \\
Liver & $204(34.2)$ \\
Bone & $74(12.4)$ \\
Lung & $48(8.1)$ \\
Other & $47(7.9)$ \\
\hline
\end{tabular}

Abbreviation: ECOG, Easter Cooperative Oncology Group.

$(8.5 \%), 175(61.6 \%)$, and $85(29.9 \%)$ received triple-, double-, or single-agent treatment, respectively. Compared with first-line chemotherapy, the proportion of patients receiving single-agent treatment was higher (29.9\% vs $10.4 \%)$. Of the 519 patients who had received first-line chemotherapy, 99 (19.1\%) subsequently received third-line treatment. Of these 99 patients, $3(3.0 \%), 59$ $(59.6 \%)$, and $37(37.4 \%)$ received triplet-, doublet-, or single-agent therapy, respectively. The first-line, secondline and third-line chemotherapy regimens are shown in Figure 1.

\section{Response}

In the first-line setting, the overall best response was complete response (CR) in $2.9 \%$, partial response (PR) in $41.4 \%$, stable disease (SD) in $29.1 \%$, and progressive disease (PD) in $26.6 \%$ (Table 2). In the second-line setting, the overall best response was a PR in $21.5 \%$, SD in $33.8 \%$, and $\mathrm{PD}$ in $44.7 \%$. In the third-line setting, the best overall response was a $\mathrm{PR}$ in $17.2 \%, \mathrm{SD}$ in $24.2 \%$, and $\mathrm{PD}$ in $58.6 \%$.

\section{Survival}

The median OS for the whole cohort from the date of diagnosis of AGC was 12.4 months (Figure 2). Survival correlated significantly with the number of treatment lines received $(\mathrm{P}<0.001$; Figure 3$)$, with a median OS from diagnosis of 3.3, 8.6, 15.6, and 21.0 months for patients receiving $0,1,2$, or $\geq 3$ lines of chemotherapy, respectively.

\section{Reasons for Chemotherapy Discontinuation}

The most frequently reported primary reason for chemotherapy discontinuation was disease progression. In first-line, second-line, and third-line chemotherapy regimens, $56.5 \%, 53.5 \%$, and $49.5 \%$ of AGC patients stopped chemotherapy due to disease progression, respectively. Other reasons for chemotherapy discontinuation included completion of chemotherapy, patient refusal, and toxicities or side effects (Table 3 ).

\section{Discussion}

Gastric cancer is the third leading cause of cancer-related death in the world. ${ }^{1}$ Most patients present with locally advanced or metastatic gastric cancer when they are first diagnosed. They have lost the opportunity for surgical intervention and can only receive medical or pharmacological treatment. ${ }^{13}$ Treatments include BSC, chemotherapy, targeted therapy, and immunotherapy. In this study, 77 patients did not receive chemotherapy and only received BSC. The median OS of these 77 patients was 3.3 months, which was consistent with many studies reporting that the median OS of patients receiving only BSC was 3-5 months. ${ }^{14}$

Chemotherapy can significantly prolong the survival of patients, and it is currently the main treatment for AGC. Le et al used the Flatiron Health database to analyze the clinical data of 3291 AGC patients and found that the median OS of patients who received first-line chemotherapy was 9.1 months. ${ }^{15}$ Trastuzumab can prolong the median OS of AGC patients to 14-16 months. However, the survival benefit of trastuzumab is limited to $15-20 \%$ of patients with HER2 overexpression. ${ }^{16,17}$ Although several first-line trials with new targeted agents have been carried 
A

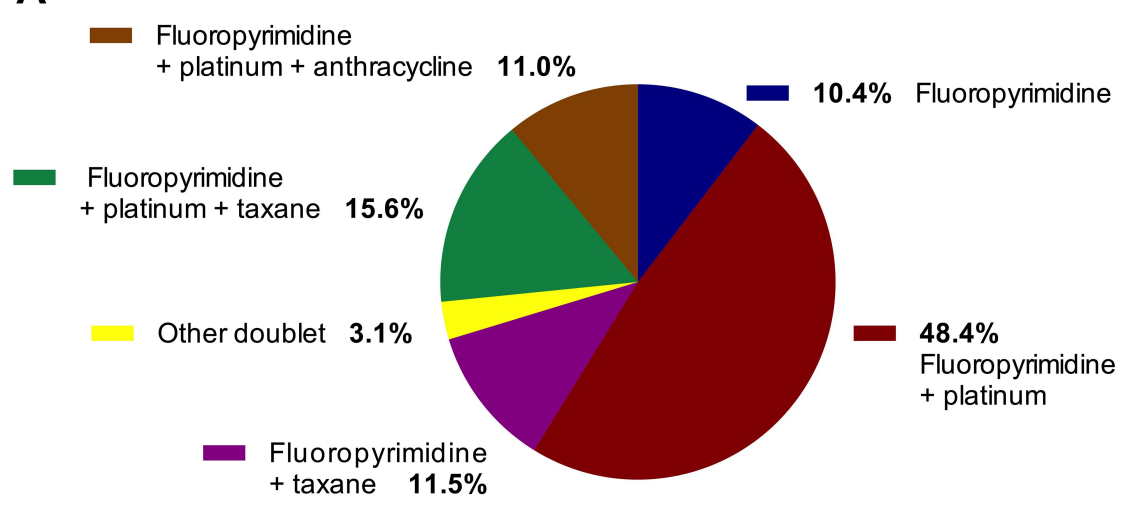

B

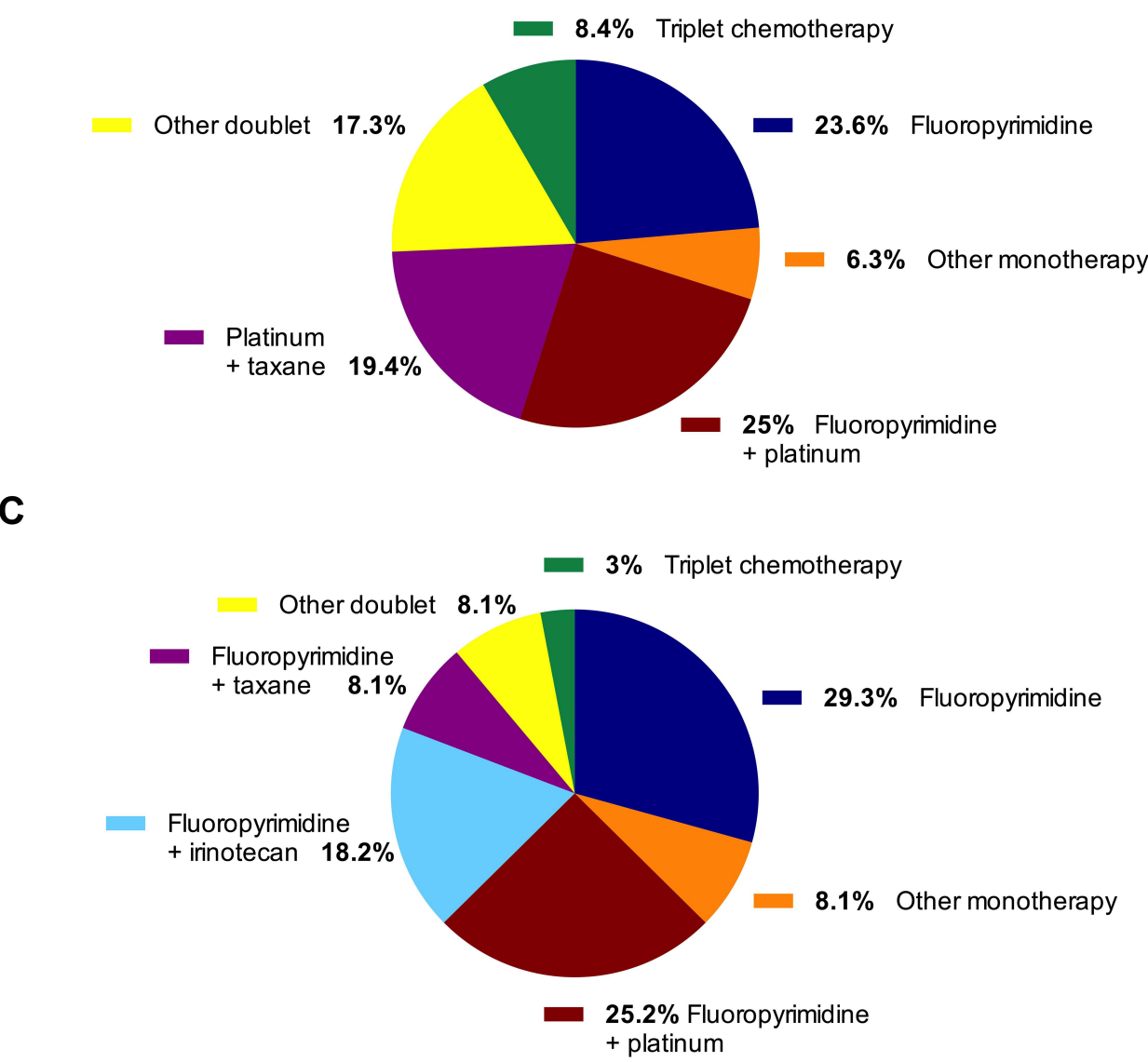

Figure I Treatment regimens received as first-line, second-line, and third-line therapy. (A) Treatments received in the first-line setting ( $\mathrm{n}=5 \mathrm{I}$ ). Other doublet treatment regimens included: Platinum + taxane $(n=9)$; Fluoropyrimidine + irinotecan $(n=7)$. $(B)$ Treatments received in the second-line setting $(n=284)$. Other monotherapy regimens included: Taxane $(n=11)$; Irinotecan $(n=7)$. Other doublet include: Fluoropyrimidine + taxane $(n=27)$; Fluoropyrimidine + irinotecan $(n=22)$. Triplet chemotherapy regimens included: Fluoropyrimidine + platinum + taxane $(n=12)$; Fluoropyrimidine + platinum + anthracycline $(n=12)$. $(\mathbf{C})$ Treatments received in the third-line setting $(n=99)$. Other monotherapy regimens included: Apatinib $(n=7)$; Taxane $(n=1)$. Other doublet regimens included: Platinum + taxane $(n=6)$; Platinum + irinotecan $(n=2)$. Triplet chemotherapy regimens included: Fluoropyrimidine + platinum + taxane $(n=2)$; Fluoropyrimidine + platinum + anthracycline $(n=I)$.

out over recent years, none has added valuable benefits. $^{18-21}$ In this study, 235 AGC patients only received 1 line of chemotherapy, and the median OS was 8.6 months. It has been reported that even the most effective first-line chemotherapy regimens are unable to achieve a median OS longer than 9-11 months. ${ }^{17}$ Since it is increasingly unlikely to improve the survival of patients with a first-line treatment only, a potential method is to expand the lines of treatment from a first- to a second- and to a third-line and beyond. ${ }^{22-24}$ 
Table 2 Variables Stratified by Treatment Line

\begin{tabular}{|l|l|l|l|}
\hline Variable & $\begin{array}{l}\text { First } \\
\text { Line }\end{array}$ & $\begin{array}{l}\text { Second } \\
\text { Line }\end{array}$ & $\begin{array}{l}\text { Third } \\
\text { Line }\end{array}$ \\
\hline Patients, n (\%) & $519(100)$ & $284(54.7)$ & $99(19.1)$ \\
\hline $\begin{array}{l}\text { Treatment, \% } \\
\text { Single }\end{array}$ & 10.4 & 29.9 & 37.4 \\
Doublet & 63.0 & 61.6 & 59.6 \\
Triplet & 26.6 & 8.5 & 3 \\
\hline Overall best response, \% & & & \\
CR & 2.9 & 0 & 0 \\
PR & 41.4 & 21.5 & 17.2 \\
SD & 29.1 & 33.8 & 24.2 \\
PD & 26.6 & 44.7 & 58.6 \\
\hline OS, months & & & \\
Whole cohort & 12.4 & & \\
According to treatment & 8.6 & $15.6(2$ & $21.0(\geq 3$ \\
line received & $(1$ line) & lines $)$ & lines $)$ \\
\hline
\end{tabular}

Abbreviations: $\mathrm{CR}$, complete response; $\mathrm{PR}$, partial response; $\mathrm{SD}$, stable disease; $\mathrm{PD}$, progressive disease; $\mathrm{OS}$, overall survival.

A Phase III clinical trial of AGC in South Korea showed that after the failure of first-line chemotherapy, the OS of patients in the salvage chemotherapy group was extended by 1.5 months compared with the BSC group $(\mathrm{P}=0.07) .{ }^{11} \mathrm{~A}$ clinical study of apatinib in the third- line treatment of AGC also showed that the median OS in the apatinib group was 1.8 months longer than that in the placebo group $(\mathrm{P}<0.001) .{ }^{10}$ The above prospective randomized clinical trials have confirmed the survival advantages of second-line or third-line chemotherapy compared with their respective control groups. In Western countries, the proportion of AGC patients receiving second-line and third-line chemotherapy is $39-42 \%$ and $14-19 \%$, respectively, ${ }^{15,16,25}$ while in Japan, the proportion is even higher, $69-85 \%$ and $23-35 \%$, respectively. ${ }^{26,27}$ This difference has important implications for the survival of AGC patients. Takashima et al found that for some clinical trials investigating first-line chemotherapy, the OS of Japanese patients with AGC was higher than non-Japanese patients, mainly because Japanese patients had longer survival after disease progression. However, due to the similarity of PFS, the survival advantage was attributed to a higher proportion of patients subsequently receiving chemotherapy in Japan. ${ }^{26}$ Iizumi et al concluded through meta-analysis that the proportion of patients receiving second-line or third-line treatment was significantly associated with the median OS of AGC patients. For every $10 \%$ increase in the proportion of patients receiving second-line or third-line treatment, the median OS could be extended by 1.033 or 0.344 months. ${ }^{28}$

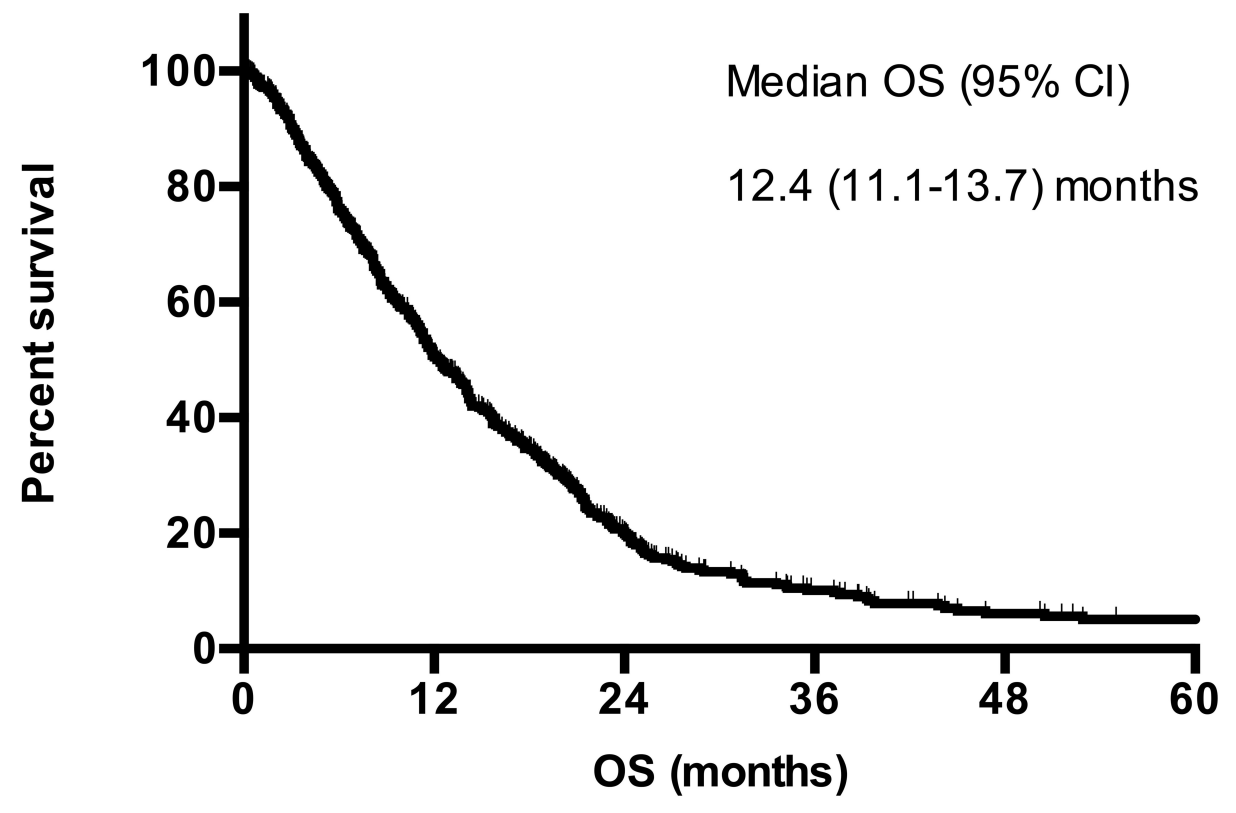

No. at risk

$\begin{array}{llllll}596 & 219 & 71 & 29 & 15 & 9\end{array}$

Figure 2 Overall survival for whole cohort.

Abbreviations: OS, overall survival; $\mathrm{Cl}$, confidence interval. 


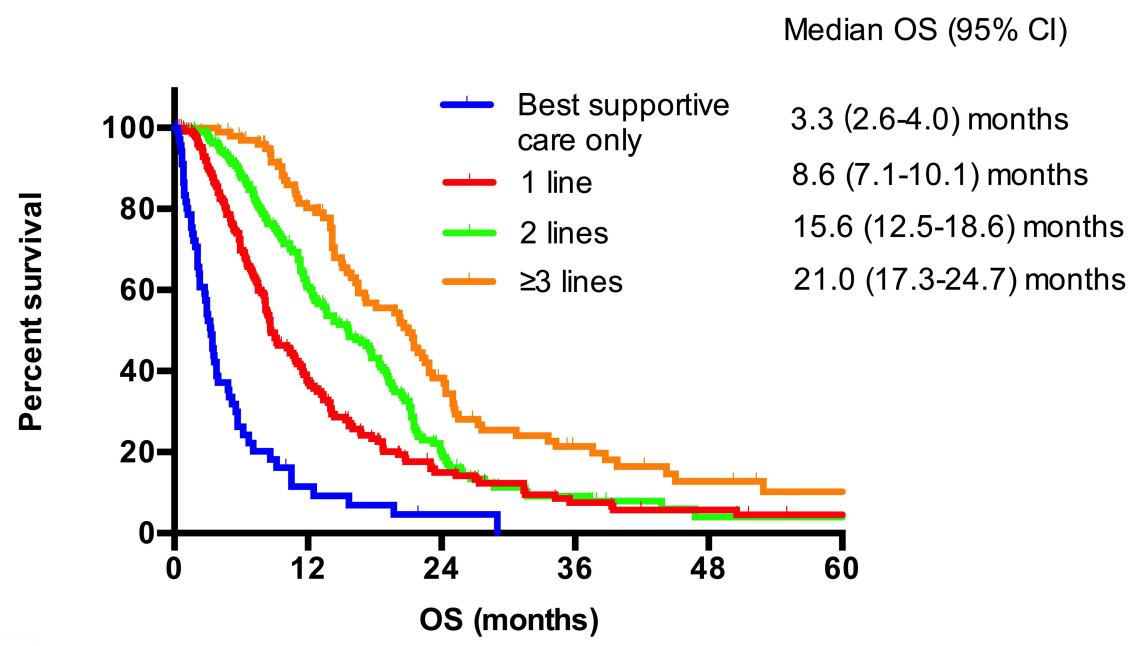

No. at risk

$\begin{array}{lllllll}\begin{array}{l}\text { Best supportive } \\ \text { care only }\end{array} & 77 & 5 & 0 & 0 & 0 & 0 \\ 1 \text { line } & 235 & 55 & 17 & 8 & 5 & 2 \\ 2 \text { lines } & 185 & 90 & 24 & 8 & 3 & 3 \\ \geq 3 \text { lines } & 99 & 69 & 30 & 13 & 7 & 4\end{array}$

Figure 3 Overall survival stratified by number of treatment lines received. Abbreviations: OS, overall survival; $\mathrm{Cl}$, confidence interval.

The above prospective clinical trials and meta-analysis confirmed the survival advantage of second-line or third-line chemotherapy. In addition, some real-world retrospective studies have also demonstrated a relationship between the number of chemotherapy lines and the survival of AGC patients. Davidson et al retrospectively analyzed the clinical data of 511 AGC patients diagnosed at the Royal Marsden Hospital in the United Kingdom from April 2009 to November 2015. The authors found that the median OS from diagnosis was 8.32, 13.95, 20.01, and 33.03 months for patients receiving $1,2,3$ or $>3$ lines of chemotherapy,

Table 3 Reasons for Chemotherapy Discontinuation

\begin{tabular}{|c|c|c|c|}
\hline $\begin{array}{l}\text { Reasons for } \\
\text { Chemotherapy } \\
\text { Discontinuation }\end{array}$ & $\begin{array}{l}\text { First Line } \\
(\mathrm{N}=5 \mid 9) \\
(\%)\end{array}$ & $\begin{array}{l}\text { Second } \\
\text { Line } \\
(\mathrm{N}=284) \\
(\%)\end{array}$ & $\begin{array}{l}\text { Third } \\
\text { Line } \\
(\mathrm{N}=99) \\
(\%)\end{array}$ \\
\hline Disease progression & 56.5 & 53.5 & 49.5 \\
\hline $\begin{array}{l}\text { Total completion of } \\
\text { chemotherapy }\end{array}$ & 15.0 & 8.8 & 8.1 \\
\hline Patient refusal & 11.9 & 11.6 & 10.1 \\
\hline Toxicities or side effects & 11.4 & 10.2 & 7.1 \\
\hline Death related to $A G C$ & 0.6 & 1.8 & 2.0 \\
\hline Patient physical status & 1.3 & 2.1 & 2.0 \\
\hline Unknown & 3.3 & 12.0 & 21.2 \\
\hline
\end{tabular}

Abbreviation: AGC, advanced gastric cancer. respectively $(\mathrm{P}<0.001) .{ }^{25}$ As the number of chemotherapy lines increased, OS was gradually prolonged. In this study, we increased the survival data of AGC patients who did not receive chemotherapy, and found that the OS of patients with chemotherapy lines of $0,1,2$ and $\geq 3$ were $3.3,8.6,15.6$, and 21.0 months, respectively $(\mathrm{P}<0.001)$. The results of this study further demonstrated the impact of the number of chemotherapy lines on OS in AGC patients. Furthermore, compared with other subgroups, patients with a number of chemotherapy lines $\geq 3$ generally tolerated treatment well and were sensitive to chemotherapy. Therefore, these patients may benefit from multi-line chemotherapy.

In addition, reasons for treatment discontinuation were also analyzed in this study. We found that due to disease progression, patient refusal, and side effects of chemotherapy, many patients stopped chemotherapy early and did not receive any subsequent anti-tumor treatment. In this study, the proportion of patients receiving second-line and third-line chemotherapy were $54.7 \%$ and $19.1 \%$, respectively. Compared with $69-85 \%$ and $23-35 \%$ of patients receiving second- and third-line chemotherapy in Japan, there is a certain gap. ${ }^{26,27}$ In clinical practice, clinicians may improve patient compliance through educational interventions, and the choice of reasonable treatment regimens, so that AGC patients may benefit from subsequent treatment. $^{29,30}$ 
However, our study also has its limitations. Firstly, this study was a single-center retrospective study, and the results may be influenced by many confounding factors; Second, the patients in this study were not homogenous, and there were differences in chemotherapy regimens used, cycles administered, and in the treatment sequences, which may have influenced the results of the study.

\section{Conclusion}

This study showed that the more chemotherapy lines AGC patients received, the longer the OS. This study not only confirmed the impact of chemotherapy lines on OS but it also supplements the results of prospective clinical trials that cannot be completed due to the ethical implications.

\section{Disclosure}

The authors report no conflicts of interest in this work.

\section{References}

1. Bray F, Ferlay J, Soerjomataram I, Siegel RL, Torre LA, Jemal A. Global cancer statistics 2018: GLOBOCAN estimates of incidence and mortality worldwide for 36 cancers in 185 countries. CA Cancer J Clin. 2018;68(6):394-424. doi:10.3322/caac.21492

2. Feng RM, Zong YN, Cao SM, Xu RH. Current cancer situation in China: good or bad news from the 2018 global cancer statistics? Cancer Commun (Lond). 2019;39(1):22.

3. Digklia A, Wagner AD. Advanced gastric cancer: current treatment landscape and future perspectives. World J Gastroenterol. 2016;22 (8):2403-2414. doi:10.3748/wjg.v22.i8.2403

4. Fujitani K, Yang H, Mizusawa J, et al. Gastrectomy plus chemotherapy versus chemotherapy alone for advanced gastric cancer with a single non-curable factor (REGATTA): a Phase 3, randomised controlled trial. Lancet Oncol. 2016;17(3):309-318. doi:10.1016/S1470-2045(15) 00553-7

5. Kondoh C, Kadowaki S, Komori A, et al. Salvage chemotherapy with the combination of oxaliplatin, leucovorin, and 5-fluorouracil in advanced gastric cancer refractory or intolerant to fluoropyrimidines, platinum, taxanes, and irinotecan. Gastric Cancer. 2018;21(6): 1050-1057. doi:10.1007/s10120-018-0825-y

6. Ford HER, Marshall A, Bridgewater JA, et al. Docetaxel versus active symptom control for refractory oesophagogastric adenocarcinoma (COUGAR-02): an open-label, phase 3 randomised controlled trial. Lancet Oncol. 2014;15(1):78-86. doi:10.1016/S1470-2045(13) 70549-7

7. Satoh T, Lee KH, Rha SY, et al. Randomized Phase II trial of nimotuzumab plus irinotecan versus irinotecan alone as second-line therapy for patients with advanced gastric cancer. Gastric Cancer. 2015;18 (4):824-832. doi:10.1007/s10120-014-0420-9

8. Ter Veer E, Haj Mohammad N, van Valkenhoef G, et al. Second- and third-line systemic therapy in patients with advanced esophagogastric cancer: a systematic review of the literature. Cancer Metastasis Rev. 2016;35(3):439-456.

9. Nishimura T, Iwasa S, Nagashima K, et al. Irinotecan monotherapy as third-line treatment for advanced gastric cancer refractory to fluoropyrimidines, platinum, and taxanes. Gastric Cancer. 2017;20 (4):655-662. doi:10.1007/s10120-016-0670-9
10. $\mathrm{Li} \mathrm{J}$, Qin $\mathrm{S}, \mathrm{Xu} \mathrm{J}$, et al. Randomized, double-blind, placebo-controlled phase III trial of apatinib in patients with chemotherapy-refractory advanced or metastatic adenocarcinoma of the stomach or gastroesophageal junction. J Clin Oncol. 2016;34 (13):1448-1454. doi:10.1200/JCO.2015.63.5995

11. Kang JH, Lee SI, Lim DH, et al. Salvage chemotherapy for pretreated gastric cancer: a randomized phase III trial comparing chemotherapy plus best supportive care with best supportive care alone. J Clin Oncol. 2012;30(13):1513-1518. doi:10.1200/JCO.2011.39.4585

12. Takashima A, Boku N, Kato K, et al. Survival prolongation after treatment failure of first-line chemotherapy in patients with advanced gastric cancer: combined analysis of the Japan Clinical Oncology group trials JCOG9205 and JCOG9912. Gastric Cancer. 2014;17 (3):522-528. doi:10.1007/s10120-013-0309-z

13. Fanotto V, Uccello M, Pecora I, et al. Outcomes of advanced gastric cancer patients treated with at least three lines of systemic chemotherapy. Oncologist. 2017;22(12):1463-1469.

14. Wagner AD, Syn NL, Moehler M, et al. Chemotherapy for advanced gastric cancer. Cochrane Database Syst Rev. 2017;8:CD004064.

15. Le DT, Ott PA, Korytowsky B, et al. Real-world treatment patterns and clinical outcomes across lines of therapy in patients with advanced/ metastatic gastric or gastroesophageal junction cancer. Clin Colorectal Cancer. 2020;19(1):32-38. doi:10.1016/j.clcc.2019.09.001

16. Smyth EC, Moehler M. Late-line treatment in metastatic gastric cancer: today and tomorrow. Ther Adv Med Oncol. 2019;11: 1758835919867522 .

17. Salati M, Di Emidio K, Tarantino V, Cascinu S. Second-line treatments: moving towards an opportunity to improve survival in advanced gastric cancer? ESMO Open. 2017;2(3):e000206. doi:10.11 36/esmoopen-2017-000206

18. Lordick F, Kang Y, Chung H, et al. Capecitabine and cisplatin with or without cetuximab for patients with previously untreated advanced gastric cancer (EXPAND): a randomised, open-label phase 3 trial. Lancet Oncol. 2013;14(6):490-499. doi:10.1016/S1470-2045(13)70102-5

19. Yoon H, Bendell J, Braiteh F, et al. Ramucirumab combined with FOLFOX as front-line therapy for advanced esophageal, gastroesophageal junction, or gastric adenocarcinoma: a randomized, double-blind, multicenter Phase II trial. Ann Oncol. 2016;27 (12):2196-2203. doi:10.1093/annonc/mdw423

20. Shah M, Bang Y, Lordick F, et al. Effect of fluorouracil, leucovorin, and oxaliplatin with or without onartuzumab in HER2-negative, MET-positive gastroesophageal adenocarcinoma: the METGastric randomized clinical trial. JAMA Oncol. 2017;3(5):620-627. doi:10.10 01/jamaoncol.2016.5580

21. Hecht J, Bang Y, Qin S, et al. Lapatinib in combination with capecitabine plus oxaliplatin in human epidermal growth factor receptor 2-positive advanced or metastatic gastric, esophageal, or gastroesophageal adenocarcinoma: TRIO-013/LOGiC-a randomized Phase III trial. J Clin Oncol. 2016;34(5):443-451. doi:10.1200/JCO.2015. 62.6598

22. Kim SM, Park SH. Chemotherapy beyond second-line in advanced gastric cancer. World J Gastroenterol. 2015;21(29):8811-8816. doi:10.3748/wjg.v21.i29.8811

23. Pan WT, Zhou SN, Pan MX, et al. Role of systemic treatment for advanced/metastatic gastric carcinoma in the third-line setting: a bayesian network analysis. Front Oncol. 2020;10:513. doi:10.33 89/fonc. 2020.00513

24. Rizzo A, Mollica V, Ricci A, et al. Third- and later-line treatment in advanced or metastatic gastric cancer: a systematic review and meta-analysis. Future Oncol. 2020;16(2):4409-4418. doi:10.2217/ fon-2019-0429

25. Davidson M, Cafferkey C, Goode EF, et al. Survival in advanced esophagogastric adenocarcinoma improves with use of multiple lines of therapy: results from an analysis of more than 500 patients. Clin Colorectal Cancer. 2018;17(3):223-230. doi:10.1016/j.clcc.2018.05. 014 
26. Takashima A, Iizumi S, Boku N. Survival after failure of first-line chemotherapy in advanced gastric cancer patients: differences between Japan and the rest of the world. Jpn J Clin Oncol. 2017;47 (7):583-589. doi:10.1093/jjco/hyx044

27. Shim HJ, Yun JY, Hwang JE, Bae WK, Cho SH, Chung IJ. Prognostic factor analysis of third-line chemotherapy in patients with advanced gastric cancer. Gastric Cancer. 2011;14(3):249-256. doi:10.1007/s10120-011-0032-6

28. Iizumi S, Takashima A, Sakamaki K, Morita S, Boku N. Survival impact of post-progression chemotherapy in advanced gastric cancer: systematic review and meta-analysis. Cancer Chemother Pharmacol. 2018;81(6):981-989. doi:10.1007/s00280-018-3569-9
29. Xie FL, Wang YQ, Peng LF, Lin FY, He YL, Jiang ZQ. Beneficial effect of educational and nutritional intervention on the nutritional status and compliance of gastric cancer patients undergoing chemotherapy: a randomized trial. Nutr Cancer. 2017;69(5):762-771. doi:10.1080/01635581.2017.1321131

30. Jang SH, Jung YJ, Kim MG, Kwon SJ. The prognostic significance of compliance with postoperative adjuvant chemotherapy in patients with stage iii gastric cancer: an observational study. J Gastric Cancer. 2018;18(1):48-57. doi:10.5230/jgc.2018.18.e4

\section{Publish your work in this journal}

Cancer Management and Research is an international, peer-reviewed open access journal focusing on cancer research and the optimal use of preventative and integrated treatment interventions to achieve improved outcomes, enhanced survival and quality of life for the cancer patient.
The manuscript management system is completely online and includes a very quick and fair peer-review system, which is all easy to use. Visit http://www.dovepress.com/testimonials.php to read real quotes from published authors. 